\title{
Upper and Lower Limits for the Number of Bound States in a Given Central Potential
}

\author{
By \\ F. Calogero \\ Istituto di Fisica dell' Università, Roma \\ Istituto Nazionale di Fisica Nucleare, Sezione di Roma
}

\begin{abstract}
Upper and lower limits for the number of bound states in a given central potential are obtained. They imply that for strongly attractive potentials the number of bound states of given angular momentum increases as the square root of the strength of the potential.
\end{abstract}

\section{Introduction}

Let $V(r)$ be a central potential such that the integral

$$
I=\int_{0}^{\infty} d r r|V(r)|
$$

is finite. Note that this integral is dimensionless in the units chosen, $\hbar=2 m=1$. Jost and PAIS [1] have shown that a necessary condition for the existence of bound states is $I \geqq 1$. Subsequently BARGManN [2], and later Schwinger [3], have derived the more general inequality

$$
n_{l} \leqq I /(2 l+1),
$$

where $n_{l}$ is the number of bound states with angular momentum $l$. They also show that the estimate eq. (1.2) is "best possible in the sense that for a given $l$ potentials may be constructed which have a prescribed number $n_{l}$ of bound states for that angular momentum and for which $I$ approaches $(2 l+1) n_{l}$ arbitrarily closely" [2]. This of course does not imply that other upper limits for the number of bound states could not be found which, for many potentials, would yield restrictions more stringent than those obtained from Bargmann's inequality eq. (1.2). In fact it should be noted that the potentials which saturate Bargmann's inequality have a rather peculiar shape; for a given $n_{l}$, they 
consist of $n_{l}$ widely separated narrow and deep wells (see Ref. [2] and $[3])^{\star}$.

In Sections 2 and 3 of this paper we derive several other rather simple upper limits for the number of bound states $\star \star$. We consider only the case of $S$-waves; of course the results hold a fortiori for higher partial waves, although the corresponding restrictions are looser. The results of Section 2 are non trivial only if the three integrals

$$
I_{p}=-\int_{0}^{\infty} d r r^{p} V(r) \theta[-V(r)], \quad p=0,1,2,
$$

are finite $(\theta(x)$ is the usual step function). The restrictions we obtain may be more or less stringent than that yielded by Bargmann's formula, depending on the shape of the potential. Some examples are given. The results of Section 3 imply that for any potential (which is finite for $r>0$ ) the number of bound states of a given angular momentum does not increase faster than the square root of the strength of the potential ${ }^{\star \star \star}$.

In Section 4 we derive a formula which gives a lower limit for the number of bound states of given angular momentum ${ }^{\dagger}$. This condition is best possible in BARGMANN's sense, and is saturated by potentials quite different in shape from those which saturate Bargmann's inequality. The results of this Section imply that the number of bound states of given angular momentum does not increase slower than the square root of the strength of the potential.

This last result, together with that mentioned at the end of the previous paragraph, implies that the number of bound states of given angular momentum increases, for strongly attractive potentials, as the square root of some measure of the strength of the potential.

For the reader who is interested only in the results, we have collected all the conditions derived in the paper in Section 5, together with the restrictions on the potentials which are required for their validity.

* Schwinger considers the case of potentials which are the sum of $n_{l} \delta$-functions [3]. A peculiarity of such potentials is that, no matter how much their strength is increased, they can never possess more than $n_{l}$ bound states for any one angular momentum (because they can bend the zero-energy radial wave function only at $n_{l}$ points). We do not take into consideration such singular potentials, except as a limiting case.

$\star \star$ Setting $n$, the number of $S$-wave bound states, equal to unity, we obtain necessary conditions which the potential must satisfy in order to be able to possess bound states at all. The condition corresponding to the results of Section 3 is identical to that given in a previous paper [4].

$\star \star \star$ This result had been previously conjectured [5].

$\dagger$ Setting the lower limit equal to one we get a sufficient condition on the potential for the existence of at least one bound state of given angular momentum. For other conditions to the same effect see Reference [6]. 


\section{Upper limits for the number of $\mathbf{S}$-wave bound states}

The number of $S$-wave bound states equals the number of poles on the positive real axis of the solution of the Riccati equation $[6-9]^{\star}$

$$
a^{\prime}(r)=-V(r)[r+a(r)]^{2},
$$

with boundary condition

$$
a(0)=0 \text {. }
$$

It is simpler to deal with a potential $V(r)$ which is everywhere attractive (negative). If the potential is repulsive in some region, it is understood that it should be replaced by the potential $V(r) \theta[-V(r)]$; this replacement makes all the results of this and the following section valid a fortiori. The potential is also required to be such that the three integrals eq. (1.3) be finite. This condition implies that the scattering length $a(\infty)$ exists and is finite ${ }^{\star \star}$.

It is now convenient to introduce after DASHEN [9] the function $\mu(r)$ through

$$
a(r)=R(\tan \mu(r)-A)
$$

where $R$ and $A$ are positive constants. We then find

$$
\mu^{\prime}(r)=R^{-1}|V(r)|[(r-R A) \cos \mu(r)+R \sin \mu(r)]^{2},
$$

with boundary condition

$$
\mu(0)=\tan ^{-1} A .
$$

Obviously $\mu(r)$ is a finite positive function, and the number $n$ of $S$-wave bound states is the integral part of $1 / 2+\mu(\infty) / \pi$. Therefore we may write

$$
n \leqq \frac{1}{2}+\frac{1}{\pi} \mu(\infty) \text {. }
$$

But from eq. (2.4) we immediately infer

$$
\mu^{\prime}(r) \leqq R^{-1}|V(r)|\left[(r-R A)^{2}+R^{2}\right],
$$

because the maximum value of $(P \cos x+Q \sin x)^{2}$ is $P^{2}+Q^{2}$. Therefore from eqs. (2.5), (2.7), (1.3) and (2.6) we conclude

$$
n \leqq \frac{1}{2}+\frac{1}{\pi} \tan ^{-1} A+\frac{1}{R \pi}\left[I_{2}-2 R A I_{1}+R^{2}\left(1+A^{2}\right) I_{0}\right] .
$$

It is now convenient to set

$$
R=\left[I_{0}\left(1+A^{2}\right) / I_{2}\right]^{-\frac{1}{2}} .
$$

* Except if the potential is such that an infinitesimal increase in its attractive strength would produce a new bound state of infinitesimal binding energy, in which case $a(r)$ has a pole at $r=\infty$. This limiting situation may be described as corresponding to the occurrence of a zero-energy bound state, because in this case the $S$ matrix has a pole at $k=0$. However the residue of this pole vanishes, and the corresponding state is not normalizable. If this situation were not considered to correspond to a bound state, then all the inequalities derived in this paper could be strengthened by eliminating the equality sign.

$\star \star$ See preceding footnote. 
We thus obtain

$$
n \leqq \frac{1}{2}+\frac{1}{\pi} \tan ^{-1} A+\frac{2}{\pi}\left\{\left[I_{0} I_{2}\left(1+A^{2}\right)\right]^{\frac{1}{2}}-A I_{1}\right\} .
$$

The choice of $A$ is still arbitrary. The most convenient one is the one which minimizes the right hand side, namely a solution of the equation

$$
A^{4}\left(I_{0} I_{2}-I_{1}^{2}\right)+A^{2}\left[I_{0} I_{2}-2 I_{1}\left(I_{1}-1\right)\right]-\left(I_{1}+1\right)^{2}=0 .
$$

Simpler choices are $A=0$, which insures

$$
n \leqq \frac{1}{2}+(2 / \pi)\left(I_{0} I_{2}\right)^{\frac{1}{2}}
$$

and $A=I_{1} /\left(I_{0} I_{2}-I_{1}^{2}\right)^{\frac{1}{2}}$, which insures

$$
n \leqq \frac{1}{2}+\frac{1}{\pi} \tan ^{-1}\left[I_{1} /\left(I_{0} I_{2}-I_{1}^{2}\right)^{\frac{1}{2}}\right]+\frac{2}{\pi}\left(I_{0} I_{2}-I_{1}^{2}\right)^{\frac{1}{2}} \text {. }
$$

From this inequality we immediately obtain the simpler expression

$$
n \leqq 1+(2 / \pi)\left(I_{0} I_{2}-I_{1}^{2}\right)^{\frac{1}{2}} \text {. }
$$

Note that $I_{0} I_{2}$ is never smaller than $I_{1}^{2}$. Thus the limitations eq. (2.12) can never improve on Bargmann's inequality by more than a factor of $(2 / \pi)$. Furthermore the expression $I_{0} I_{2}-I_{1}^{2}$ can never become negative; it vanishes only for a delta-like potential $V(r)=-\left(I / r_{0}\right) \delta\left(r-r_{0}\right)$. Therefore eq. (2.14) implies that such a potential can support at most one $S$-wave bound state, irrespective of its strength $I$. On the other hand Bargmann's condition, eq. (1.2), tells us that the number of bound states is smaller than $I$. Thus for $I>1$ the condition eq. (2.14) is more stringent than Bargmann's inequality, and viceversa for $I<1$. The value $I=1$, at which the two conditions coincide, is also the exact limiting value for the appearance of one bound state. We thus see that, at least for $n=1$, the condition eq. (2.14) is also best possible in Bargmann's sense and is saturated by the same type of potential which saturates Bargmann's inequality.

For a square well potential of range $r_{0}$ and depth $\left|V_{0}\right|$, eq. (2.12) gives $n \leqq 1 / 2+(2 /(\pi \sqrt{3}))\left|V_{0}\right| r_{0}^{2}$ and eq. (2.14) gives $n \leqq 1+(1 /(\pi \sqrt{3}))$ $\left|V_{0}\right| r_{0}^{2}$, while the Bargmann condition gives $n<(1 / 2)\left|V_{0}\right| r_{0}^{2}$. Thus for large values of $\left|V_{0}\right| r_{0}^{2}$ both restrictions, and especially the second one, are more stringent than Bargmann's inequality. However for large values of $\left|V_{0}\right| r_{0}^{2}$ these conditions yield rather poor upper limits to the number of bound states; in fact the exact relationship is $n \leqq 1 / 2+$ $+(1 / \pi)\left(\left|V_{0}\right| r_{0}^{2}\right)^{\frac{1}{2}}$, which implies that the number of bound states increases as the square root of the potential rather than linearly. This is a general property, valid for all potentials, as is shown in the following section. 


\section{Another upper limit for the number of $S$-wave bound states}

The result of this section consists of a generalization to the case of $n$ bound states of the condition proved in Reference [4] for the case of one bound state. The generalization is easily accomplished with an argument similar to one used by BargmanN [2].

In this Section we assume the potential to be monotonic. Since the potential must vanish at infinity and be attractive, this implies the condition

$$
V^{\prime}(r) \geqq 0 \text {. }
$$

Only for potentials of this type holds the result which we are going to prove. However given any potential $V(r)$ it is always possible to find a monotonic potential $\bar{V}(r)$ such that $\bar{V}(r) \leqq V(r)$; and the upper limit which we could obtain for the number of bound states of $\bar{V}(r)$ would hold a fortiori for the number of bound states of $V(r)$.

Let us now assume that the potential $V(r)$ possesses $n S$-wave bound states. This implies that the function $a(r)$ introduced in the preceding Section has $n$ poles on the positive real axis, at the points $r=p_{i}$, $i=1,2, \ldots, n$. It is also apparent from the structure of the Riccati equation satisfied by $a(r)$, eq. (2.1), that $a(r)$ also has $n$ zeros, at the points $r=z_{i}, i=1,2, \ldots, n$ (with $z_{1}=0$ and $z_{i}<p_{i}<z_{i+1}$ ). We now define

and

$$
J_{i}=\int_{z_{i}}^{p_{i}} d r|V(r)|^{\frac{1}{2}}
$$

$$
J=\int_{0}^{\infty} d r|V(r)|^{\frac{1}{2}} .
$$

Obviously

$$
J \geqq \sum_{i=1}^{n} J_{i} .
$$

But the result proved in Reference (4) implies that

We therefore conclude that

$$
J_{i} \geqq \pi / 2 \text {. }
$$

$$
J \geqq n \pi / 2 \text {, }
$$

or equivalently,

$$
n \leqq(2 / \pi) \int_{0}^{\infty} d r|V(r)|^{\frac{1}{2}} .
$$

This is the required upper limit on the number of bound states.

It should be noted that this result implies that the number of bound states for a given angular momentum increases at most as the square root of the strength of the potential (coupling constant). Note that this statement holds for any potential ${ }^{\star \star}$, not only for those satisfying the restriction eq. (3.1), as indicated in the remark following that equation.

$\star$ We exclude potentials which are singular for $r>0$.

$\star \star$ See preceding footnote. 


\section{Lower limit for the number of bound states of given angular momentum $l$}

The number of bound states $n_{l}$ of angular momentum $l$ coincides with the number of poles on the positive real axis of the functions $a_{l}(r)$, defined by the Riccati equations $[8,9]$

$$
a_{l}^{\prime}(r)=-(2 l+1)^{-1} \frac{V(r)}{q}(q r)^{-2 l}\left[(q r)^{2 l+1}+a_{l}(r)\right]^{2},
$$

with boundary condition

$$
a_{l}(0)=0 \text {. }
$$

Here $q$ is an arbitrary constant, with the dimension of an inverse length.

It is convenient to introduce a new function $f(r)$ through

$$
(q r)^{2 l+1}+a_{l}(r)=(2 l+1) \tan f(r) .
$$

It is then clear that

$$
n_{l} \geqq\left\{\left\{\frac{1}{2}+(f / \pi)\right\}\right\}
$$

where the symbol $\{\{\}\}$ means the integral part of the quantity in the curly backets, and

$$
f=\lim _{r \rightarrow \infty} f(r) .
$$

We are assuming here that this limit exists, which is the case if the potential vanishes asymptotically faster then $r^{-2 l-3}$; but it is easily seen that our final result holds even for potentials which violate this condition.

On the other hand the function $f(r)$ satisfies the differential equation

$$
f^{\prime}(r)=q(q r)^{2} \imath \cos ^{2} f(r)-\frac{V(r)}{q}(q r)^{-2} \imath \sin ^{2} f(r),
$$

with boundary condition

But eq. (4.6) implies that

$$
f(0)=0
$$

$$
f^{\prime}(r) \geqq \operatorname{Min}\left[q(q r)^{2} l,-\frac{V(r)}{q}(q r)^{-2} l\right],
$$

because the minimum value of $A \cos ^{2} x+B \sin ^{2} x$ is the smaller number between $A$ and $B$. From this equations and eqs. (4.7), (4.5) and (4.4) we obtain our final result, namely

$$
n_{l} \geqq\left\{\left\{\frac{1}{2}+\frac{1}{\pi} \int_{0}^{\infty} d r \operatorname{Min}\left[q(q r)^{2} l,-\frac{V(r)}{q}(q r)^{-2} l\right]\right\}\right\} .
$$

It should be emphasized that in this equation the symbol $\operatorname{Min}(A, B)$ is defined as follows:

$$
\begin{aligned}
\operatorname{Min}[A, B] & =A \quad \text { if } \quad A \leqq B \\
& =B \text { if } \quad B \leqq A
\end{aligned}
$$


while the constant $q$ is arbitrary. In the case of a monotonic (attractive) potential we may rewrite eq. (4.9) as follows:

$$
n_{l} \geqq\left\{\left\{\frac{1}{2}+\frac{\left(q r^{*}\right)^{2 l+1}}{\pi(2 l+1)}+\frac{1}{\pi} \int_{r^{*}}^{\infty} d r \frac{|V(r)|}{q}(q r)^{-2 l}\right]\right\},
$$

where $r^{*}$ is the (unique) solution of the equation

$$
q^{2}\left(q r^{*}\right)^{4} \iota+V\left(r^{*}\right)=0
$$

For $S$-waves and for potentials which satisfy the condition

$$
V(r) \geqq-M^{2}
$$

with $M$ finite, we may set $q=M$ in eq. (4.9) and we obtain the very simple equation

$$
n_{0} \geqq\left\{\left\{\frac{1}{2}-\int_{0}^{\infty} d r V(r) /(\pi M)\right\}\right\}
$$

But this is not the most profitable choice for $q$. For instance for an expotential potential $V(r)=-\left|V_{0}\right| \exp \left(-r / r_{0}\right)$ eq. (4.14) yields

$$
n_{0} \geqq\left\{\left\{\frac{1}{2}+r_{0}\left|V_{0}\right|^{\frac{1}{2}} / \pi\right\}\right\}
$$

while with the optimal choice $q=\left|V_{0}\right| / e$ we derive from eqs. (4.9) or (4.11) the more stringent condition

$$
n_{0} \geqq\left\{\left\{\frac{1}{2}+2 r_{0}\left|V_{0}\right|^{\frac{1}{2}} /(\pi \sqrt{e})\right\}\right\} \text {. }
$$

From this inequality we find that to secure respectively one and two bound states it is sufficient that the parameter $r_{0}^{2}\left|V_{0}\right|$ exceeds 1.67 resp. 15 . The exact minimum values of $\left|V_{0}\right| r_{0}^{2}$ are 1.44 and 6.1 , respectively.

It is easily seen that the potential

$$
\begin{aligned}
V_{l}(r) & =-Q^{2}(Q r)^{4 \imath} & & r<r_{0} \\
& =0 & & r>r_{0}
\end{aligned}
$$

saturates the condition (4.9); that is, it is possible to adjust $Q$ and $r_{0}$ so that $V_{l}(r)$ possesses $n_{l}$ bound states of angular momentum $l$ and is such that, when substituted in the r.h.s. of equation (4.9) (with $q=Q$ ), it yields a value for the quantity within the curly backets which is arbitrarily close to $n_{l}$. We may therefore assert that the condition (4.9) is best possible in BARGMANN's sense. Note that for $S$-waves the saturating potential is a square well. It is remarkable that this potential is just of the opposite shape to the potential which saturates Bargmann's inequality and the inequality eq. (2.14); here the potential is spread out evenly over its whole range, there it is all concentrated at one point. 
Finally we note that the lower limit to the number of bound states given by the inequality (4.9) implies that this number must increase at least as the square root of the strength of the potential. This is immediately seen choosing the arbitrary parameter $q$ proportional to $g$ raised to the power $[2(2 l+1)]^{-1}$, where the "coupling constant" $g$ is some measure of the strength of the potential*.

\section{Summary of results}

Let $n_{l}$ be the number of bound states of angular momentum $l$ in the central potential $V(r)$. Of course

$$
n_{l} \leqq n_{0} \equiv n,
$$

so that any upper limit on $n$ holds a fortiori for $n_{l}$.

We have shown in Section 2 that

$$
n \leqq \frac{1}{2}+(2 / \pi)\left(I_{0} I_{2}\right)^{\frac{1}{2}},
$$

and also

$$
n \leqq 1+(2 / \pi)\left(I_{0} I_{2}-I_{1}^{2}\right)^{\frac{1}{2}} .
$$

The quantities $I_{p}$ are defined by

$$
I_{p}=-\int_{0}^{\infty} d r r^{p} V(r) \theta[-V(r)], \quad p=1,2,3,
$$

where $\theta(x)$ is the usual step function. More stringent, but also more complicated restrictions are given by eqs. (2.10) and (2.13). Of course these conditions are nontrivial only if the three integrals $I_{p}$ are finite. Note that these upper limits are linear in the potential strength, just as in the Bargmann-Schwinger condition

$$
n_{l} \leqq I_{1} /(2 l+1) .
$$

We have shown in Section 3 that

$$
n \leqq(2 / \pi) \int_{0}^{\infty} d r|V(r)|^{\frac{1}{2}}
$$

provided the potential $V(r)$ is monotonic, i.e.

$$
V^{\prime}(r) \geqq 0 \text {. }
$$

This condition implies that the number of bound states of a given angular momentum does not increase faster than the square root of the strength of the potential. This conclusion holds for all potentials $\star \star$, because it is always possible to find a monotonic potential which minorizes them.

* Note that in the limit when the potential becomes a delta function (or a finite sum of $N$ delta functions) the integral in eq. (4.9) vanishes. In fact, as we already noted, such potentials have the peculiarity that the number of bound states never increases beyond $N$.

$\star \star$ We exclude potentials which are singular for $r>0$. 
We have shown in Section 4 that

$$
n_{l} \geqq\left\{\left\{\frac{1}{2}+\frac{1}{\pi} \int_{0}^{\infty} d r \operatorname{Min}\left[q(q r)^{2} l,-\frac{V(r)}{q}(q r)^{-2} l\right]\right\}\right\},
$$

where the symbol $\{\{\}\}$ stands for integral part, the symbol Min is defined by

$$
\begin{aligned}
& \operatorname{Min}[A, B]=A \quad \text { if } \quad A \leqq B \\
& =B \text { if } B \leqq A \text {, }
\end{aligned}
$$

and $q$ is an arbitrary constant with the dimensions of an inverse length. This condition is best possible in Bargmann's sense, and is saturated by the type of potentials given in eq. (4.17). For $S$-waves and potentials satisfying the condition

$$
V(r) \geqq-M^{2}
$$

with $M$ finite, we also have the weaker but much simpler condition

$$
n \geqq\left\{\left\{\frac{1}{2}-\frac{1}{\pi} \int_{0}^{\infty} d r V(r) / M\right\}\right\} .
$$

This condition is also best possible in BARGMANN's sense, and is saturated by a square well potential.

These lower limits on the number of bound states imply that $n_{l}$ cannot increase slower then the square root of some measure $g$ of the strength of the potential (Proof: take $q$ proportional to $g^{-[2(2 l+1)]^{-1}}$ in eq. (5.7)). We may therefore conclude that asymptotically the number of bound states becomes proportional to the square root of the strength of the potential

$$
n_{l} \sim \sqrt{g} .
$$

\section{References}

[1] Jost, R., and A. PaIs: Phys. Rev. 82, 840 (1951).

[2] Bargmann, V.: Proc. Acad. Sci. USA 38, 961 (1952).

[3] Schwinger, J.: Proc. Acad. Sci. USA 47, 122 (1961).

[4] Calogero, F.: Nuovo Cimento 36, 199 (1965).

[5] SpRuch, L.: Minimum principles in scattering theory. In: Lectures in Theoretical Physics. Edited by W. E. Britrin, B. W. Downs, and J. Downs. Vol. IV., p. 198. New York: Interscience Publishers 1962.

[6] Calogero, F.: J. Math. Phys. 6, 161 (1965) and to be published.

[7] - Nuovo Cimento 27, 261 (1963).

[8] Levy, B. R., and J. B. Keller: J. Math. Phys. 4, 54 (1963).

[9] Dashen, R. F.: J. Math. Phys. 4, 388 (1963). 